\title{
Apical Organization in the Somatic Hyphae of Fungi
}

\author{
By W. KATHARINE MCCLURE, D. PARK AND P. M. ROBINSON \\ Botany Department, Queen's University, Belfast
}

(Accepted for publication I July 1967)

\begin{abstract}
SUMMARY
By using phase-contrast microscopy a 'Spitzenkörper' was seen at the hyphal apex of all the 'septate' fungi examined, but none was seen at the apex of any Phycomycete. The spitzenkörper accepts staining with cationic dyes. A method is described for consistently obtaining ultra-thin sections longitudinally through hyphal apices. Electron micrographs indicate that the spitzenkörper is an aggregation of vesicles just beyond the apex, namely at the region where wall-formation occurs during extension growth. It is suggested that the vesicles are formed posteriorly and migrate to the apex where they fuse with the plasma membrane liberating their contents as part of the process of wall growth.
\end{abstract}

\section{INTRODUCTION}

During an investigation of nuclear behaviour and mating strains in the genus Coprinus Brunswik (I924) examined iron-haemotoxylin stained preparations of two species (C. sterquilinus, C. narcoticus) and observed their hyphal tips to contain a strongly staining area at the growing point. This area he described as a 'nucleuslike' body, but because of its variable size and because he never observed it in the process of division, he concluded that it was not a nucleus but a special organelle, connected in some way with apical growth of the hypha; from its position he called it the 'Spitzenkörper'. Girbardt (I955) showed that this structure might be observed in living material by phase-contrast microscopy, and he detected its presence in the tips of several species of Basidiomycetes and in species of Penicillium and Aspergillus. He also noted that it disappeared when extension growth ceased and formed again just before extension growth restarted in a checked tip. Girbardt later (1957) used $14-16 \%(\mathrm{w} / \mathrm{v})$ gelatin in the growth medium to match the refractive index of the immersion medium with that of the fungal cytoplasm and was able to make a more detailed study of the relationship between apical growth and the behaviour of the spitzenkörper. The position of the structure in the apical dome was related to subsequent direction of extension, an eccentric position preceding a turning of the tip. No information was obtained about the source or the nature of the spitzenkörper.

Little is known of the mechanism of wall extension at the hyphal apex during growth of fungi, yet the nature of the wall here and the mechanism of its extension have considerable significance for fungal morphogenesis. The present report arises out of a study of fungal fine structure in its relation to morphogenesis.

Vol. 50, No. I was issued 7 February 1968 


\section{METHODS}

The fungi examined most frequently in this work were Aspergillus niger v. Tiegh., Fusarium oxysporum Sny. et Hans., and $F$. solani Sny. et Hans. The nutrient medium used for growth was: glucose, 0.7 g. ; $\mathrm{KH}_{2} \mathrm{PO}_{4}, 0 \cdot 2$ g.; $\mathrm{MgSO}_{4} \cdot 7 \mathrm{H}_{2} \mathrm{O}, 0.5 \mathrm{~g}$.; $\mathrm{NH}_{4} \mathrm{NO}_{3}$, $0.1 \mathrm{~g}$; ; distilled water to I 1.; agar media contained Io g. Oxoid no. 3 agar/1. Cultures were incubated for at least 2 days at $25^{\circ}$ to allow apices to attain a mature condition.

For material to be fixed and stained for light microscopy and electron microscopy sterile cover glasses were prepared with a thin $(0.5 \mathrm{~mm}$.) layer of agar medium. These were point-inoculated with spores of the fungus, and incubated on moist sterile filter papers in a humid chamber. Fixation for light microscopy was by freezesubstitution in absolute ethanol at $-70^{\circ}$, the fixed material being allowed slowly to reach $4^{\circ}$ in the ethanol.

For phase-contrast observations of growing hyphae the nutrient medium was made up in $25 \%(\mathrm{w} / \mathrm{v})$ acacia gum (Barer \& Joseph, 1958) and the fungus mounted for microscopy in the same medium, the refractive index of which is close to that of the cytoplasm (Park \& Robinson, 1957). The thin-layer cultures to be used for electron microscopy were made on coverslips which had previously been sprayed lightly with PTFE (polytetrafluoroethylene). The cultures were incubated for 3 days at $25^{\circ}$ before treatment. The coverslips with colony and medium attached were then immersed for $\mathrm{I} \mathrm{hr}$ in $2.5 \%(\mathrm{w} / \mathrm{v})$ glutaraldehyde at $0^{\circ}$, followed by washing for three $30 \mathrm{~min}$. periods in $0.1 \mathrm{M}$-phosphate buffer $\left(\mathrm{pH} 7^{\circ} 0\right)$. After post-fixation for $2 \mathrm{hr}$ with $2 \%$ osmium tetroxide in $0^{\prime} \mathrm{I} \mathrm{M}$-phosphate buffer $(\mathrm{pH} 7 \cdot 0)$, the tissue was dehydrated by passing it through a graded series of acetone solutions kept at $0^{\circ}$ at $30 \mathrm{~min}$. intervals, finally transferring to anhydrous acetone for not less than $2 \mathrm{hr}$. A I: $\mathrm{I}$ Araldite:anhydrous acetone mixture was added over a period of $\mathrm{I} h \mathrm{hr}$ until the mixture immersing the tissue became near I: I resin:acetone. The tissue was allowed to remain in this mixture for $3 \mathrm{hr}$ and then the acetone was allowed to evaporate for $24 \mathrm{hr}$, residual acetone being removed in a vacuum oven at $60^{\circ}$ and a pressure of $5 \times 10^{-3} \mathrm{~mm} . \mathrm{Hg}$.

The preparation was embedded in Araldite by using a modification of the clamped holder first described by Sheffield (1965) with slight modification. BEEM capsules (supplied by LKB Instruments Ltd.) trimmed to remove the frustrum of the hexagonal pyramid were put into the holders, and the coverslips placed on top so that the margin of the growing colony lay across the diameter of the capsule. A glass slide was then placed on top of the coverslips, the compression plate put on top of the glass slide and the whole assembly carefully bolted into position. The holder was then inverted and the capsules filled with resin. In this way the thin agar layer containing the hyphal tips was embedded and also initially orientated so that the hyphae were parallel with the flat lower exposed face of the polymerized cylinder. After the normal polymerization process the compression plate and the glass slide were removed and the coverslips gently pulled away from the capsules. The PTFE coating made this possible. The capsules were then removed from the holder.

To determine the orientation of the hyphae the blocks were examined under a binocular microscope. The block was then trimmed so that when placed in the microtome the hyphae lay with their long axes parallel to the knife edge. Sections cut with a diamond knife and a Porter-Blum microtome were placed on platinized grids, 
stained with uranyl acetate and Reynold's lead citrate, and stabilised with a thin layer of carbon.

Sections were examined in an A.E.I. EM6B making use of $250 \mu$ condenser lens aperture, $50 \mu$ objective lens aperture and cold finger to prevent specimen contamination. The beam acceleration voltage was $60 \mathrm{kV}$ and the beam current was $200 \mu$. The recording emulsion was Ilford $\mathrm{N} 40$.

\section{RESULTS}

\section{Phase-contrast observations of living hyphae}

The method used gave very good optical resolution of cytological detail by eliminating the lens effect of the narrow cylindrical hyphae. Nuclei appeared bright, each delimited by a dense boundary. Each nucleus had an opaque nucleolus situated to one side. Vacuoles (not present at the tip) were transparent and occasionally contained dense granules showing random erratic movement. The boundaries of the vacuoles were not rigidly fixed but constantly undulated, and not infrequently the vacuolar membrane invaginated into the vacuole or evaginated into the cytoplasmic matrix. These observations suggest that there was no marked pressure difference between the vacuole and the cytoplasmic matrix. The mitochondria were optically dense, filamentous, and in the tip region of the hyphae densely aggregated into a tuft. They moved actively with a serpentine motion. Small, highly refractive spherical organelles, in some cases actively moving, in other cases static, were present. These corresponded closely with the Woronin bodies described by Buller (1933). All these structures appeared clearly and with a sharp outline. The 'Spitzenkörper', on the other hand, while clearly evident could not be focused sharply even though it showed no obvious rapid movement (Pl. I, fig. I). Its boundaries were vague. Commonly associated with, and just posterior to, the spitzenkörper were two or three of the Woronin bodies moving fairly rapidly with an apparent random motion but keeping more or less the same station, not frequently traversing as did some of the other refractive bodies elsewhere. At intervals a refractive spherical body from a region posterior to the tip was seen to move forwards and take up a position behind the spitzenkörper, or from a position at the apex move steadily posteriorly.

Our observations support those of Girbardt in that the spitzenkörper is only visible during active extension growth of the hyphal tip. When growth was stopped by any of a number of agencies (poor aeration, hypertonic solution, vacuolation factor) the spitzenkörper faded out gradually. It was not seen to move away. Some of the agents used, e.g. hypertonic solutions, had only a temporary inhibitory effect and the spitzenkörper re-appeared before growth became evident once more.

We have examined several species of fungi other than the three named earlier. The list in Table I shows that a spitzenkörper was seen in every imperfect fungus examined, but in none of the Zygomycetes or Oomycetes. In Rhizopus nigricans some especially favourable preparations showed an area just behind the apex and slightly larger than the usual spitzenkörper, with small, just resolvable, particles in highly active movement. We do not consider the range of fungi examined large enough to warrant any generalization.

In material fixed by freeze-substitution in ethanol at $-70^{\circ}$ the spitzenkörper stained with methyl green + pyronin (Brachet, 1953) and with other cationic dyes (Pl. I, 
fig. 2). However, it gave this reaction even after prolonged incubation in RNAase solution so that it seems unlikely that the reaction is one for RNA. The stains used did not give a DNA reaction; the nucleus remained uncoloured whereas the nucleolus stained densely.

Table I. Fungi examined for presence of Spitzenkörper

$\begin{array}{lc} & \begin{array}{c}\text { Presence of } \\ \text { Spitzenkörper }\end{array} \\ \text { Pythium mamillatum Meurs. } & - \\ \text { Pythium ultimum Trow. } & - \\ \text { Mucor hiemalis Wehm. } & - \\ \text { Zygorhynchus moelleri Vuill. } & - \\ \text { Rhizopus' stolonifer (Ehrenb. ex. Fr.) Lind. } & - \\ \text { Aspergillus niger v. Tiegh. } & + \\ \text { A. oryzae (Ahlb.) Cohn } & + \\ \text { Botrytis cinerea Pers. ex Fr. } & + \\ \text { Fusarium culmorum (W. G. Smith) Sacc. } & + \\ \text { F. oxysporum Schlect. sensu Sny. \& Hans. } & + \\ \text { Fe solani (Mart.) App. \& Wollenw. sensu Sny. \& Hans. } & + \\ \text { Penicillium citrinum Thom } & + \\ \text { P. expansum Link. emend Thom } & + \\ \text { Trichoderma viride Pers. ex Fr. } & + \\ & +\end{array}$

\section{Electron microscopy}

The primary interest of this work is the special organization near the hyphal apex and the following description is restricted to those points of interest especially relevant to this region. The cell wall over the apex showed little structural difference from that seen in other regions of the hyphae, being largely electron transparent, and continuous over the whole apical dome in all sections examined. The wall over the dome was in some sections thicker (about 50-60 $\mathrm{m} \mu$ ) than the wall along the tubular part of the hyphae (about $30 \mathrm{~m} \mu$ ) (Pl. 2, fig. 4). This may, at least in part, have been due to some of the sections being truly longitudinal but not truly median, and therefore not cutting the wall normal to the surface.

Mitochondria in this region were filamentous, having a diameter as little as $\mathbf{I} 20 \mathrm{~m} \mu$ but usually $220-250 \mathrm{~m} \mu$. Their length was difficult to determine accurately because of their sinuous form, but lengths of $3.5 \mu$ were common, and lengths of up to $4.5 \mu$ were seen in individual sections near the tip. Mitochondria 5-7.5 $\mu$ long are common in phase-contrast photomicrographs. Mitochondria near the hyphal tip characteristically had a high content of cristae, which were tubular and orientated parallel to the long axis. A significant characteristic of the mitochondrial tuft at the tip was that it invariably stopped short (about $\mathrm{I}-2 \mu$ ) of the apex. Mitochondria were thus excluded from the area immediately behind the apical dome.

Similarly, ribosome groups, whose distribution was dense in the tip region, were infrequent in the region immediately behind the extending apex proper (PI. 3, fig. 5). Occasional ribosomes were found at the apex but were much less common and less commonly associated into groups. Nuclei were never found closer to the apex than $400 \mu$.

The unit membrane-bound vesicles immediately behind the apical dome are of interest. They appeared in all longitudinal sections of apices in extension growth at 
the time of fixation. The vesicles varied in size from $70 \mathrm{~m} \mu$ to $\mathrm{I} 20 \mathrm{~m} \mu$ and electrondense strands or particles were included in them. Similar vesicles were occasionally present in regions of hyphae proximal to the tip (Pl. 4, fig. 6), but not in the same frequency as at the apex. The region where these vesicles were most numerous corresponds with that occupied by the spitzenkörper, and was the region of exclusion of mitochondria and of ribosome groups.

The apices of lateral branches show exactly the same features as the leader hyphae from the colony margin (P1. I, fig. 3). This figure shows the presence of vesicles like those in the apical aggregate near the junction of the branch with the main axis. Zalokar (1959) has demonstrated that the normal rate of extension of an apex of a filamentous fungus is dependent on the presence of a substantial amount of subtending hyphae, and Butler (I96I) has shown a correlative situation between the extension rates of leader hyphae and its branches. Competition between apices for a common supply from posterior hyphal regions may be responsible for this correlative growth pattern.

A fusion of vesicles with the unit-membrane of the plasmalemma was clearly evident (Pl. 4, fig. 6; Pl. 3, fig. 5). It is always difficult to interpret the direction of any dynamic movements in living systems from fixed material. It seems probable that the spitzenkörper is a presentation of the mass of aggregated vesicles. Supporting this is the fact that sections from apices, the extension growth of which had been inhibited before fixation, showed no similar aggregation of vesicles at the apex. The staining with cationic dyes might indicate that the contents included acid carbohydrates. We suggest that the vesicles arise proximal to the apex and move forward, eventually to fuse with the plasmalemma, their contents being concerned with cell-wall synthesis. We have no evidence as to the source of the vesicles or to the site of their formation. No Golgi apparatus was seen in any of our sections of the three species examined by this technique. The vesicles may be produced in the region of dense mitochondria and ribosomal groups, but in the absence of information on their possible rates of migration their distribution does not exclude other origins.

Other structures regularly found in the region just behind the apical dome were the larger spherical unit membrane-bound structures (I40-3 10 $\mathrm{m} \mu$ ) with electron-dense contents (Pl. 3, fig. 5). These appeared very similar to the Woronin bodies which we found near septa in more mature regions; there were often two to four near the apex.

We wish to thank the Medical Research Council for financial support, and Professor D. J. Carr for help in preparing the manuscript. We are indebted to $\mathrm{Mr} \mathrm{R}$. Reed of the Electron Microscopy Unit, Queen's University of Belfast, for his assistance.

\section{REFERENCES}

BARER, R. \& JOSEPH, S. (1958). Concentration and mass measurements in microbiology. J. appl. Bact. 21, 146.

BracheT, J. (I935). The use of basic dyes and ribonuclease for the cytochemical detection of ribonucleic acid. Q. J. microscop. Sci. 94, 1 .

BrunswiK, H. (1924). Untersuchungen über die Geschlechts- und Kernverhältnisse bei den Hymenomyceten gattung Coprinus. Bot. Abh. K. Geobel. Heft. 5.

Buller, A. H. R. (1933). Researches on Fungi, Vol. 5. London: Longmans, Green and Co.

Butler, G. M. (1961). Growth of hyphal branching systems in Coprinus disseminatus. Ann. Bot. 25, 34I. 
Girbard, M. (1955). Lebendbeobachtungen an Polystictus versicolor (L). Flora, Jena 142, 540.

Girbardt, M. (1957). Der Spitzenkörper von Polystictus versicolor (L). Planta 50, 47.

PARK, D. \& ROBINSON, P. M. (1967). A fungal hormone controlling internal water distribution normally associated with 'cell' ageing in fungi. Symp. Soc. exp. Biol. 21, 323.

Sheffield, H. G. (1965). A device for flat-face, epoxy resin embedding of tissues and coverslip cultures. Stain Technl. 40, I43.

ZaloKAR, M. (1959). Growth and differentiation of Neurospora hyphae. Am. J. Bot. 46, 602.

\section{EXPLANATION OF PLATES}

\section{Plate I}

Fig. I. Fusarium oxysporum. Hyphal tip growing in $25 \%$ (w/v) gum acacia solution. Filamentous mitochondria, refractive spherical bodies (Woronin bodies?) and the spitzenkörper are visible. Note the tuft of mitochondria just back of the apex. No nuclei are present this near the apex. Phase contrast. $\times 2000(625 \times 3 \cdot 2)$.

Fig. 2. Aspergillus niger. Fixed hyphal tip stained methyl green + pyronin. Phase contrast. $\times 2000$ $(625 \times 3 \cdot 2)$.

Fig. 3. Aspergillus niger. Longitudinal section of lateral branch and main axis. The apex is organized in the same way as a leader apex. Electron micrograph, glutaraldehyde-O $\mathrm{SO}_{4} \cdot \times 10,875(7500 \times \mathrm{I} \cdot 45)$

\section{Plate 2}

Fig. 4. Aspergillus niger. Longitudinal section of hyphal apex. The unit membrane-bound vesicles (Vs) constituting the spitzenkörper contain an electron-transparent cortical region surrounding an electrondense core. $\mathrm{M}$, mitochondrion; $\mathrm{R}$, ribosome groups; $\mathrm{P}$, plasma membrane, $\mathrm{CW}$, cell wall. Electron micrograph, glutaraldehyde-OsO $\mathrm{O}_{4} \times 72,000(20,000 \times 3 \cdot 6)$.

\section{Plate 3}

Fig. 5. Aspergillus niger. Longitudinal section of hyphal tip. Two Woronin bodies (W) appear in this section. The configuration of the plasma membrane $(\mathrm{P})$ at the apex suggests fusion of the vesicles (Vs) with the membrane. M, mitochondria; R, ribosome groups. Electron micrograph, glutaraldehyde$\mathrm{OsO}_{4} \times 32,000(10,000 \times 3 \cdot 2)$.

\section{Plate 4}

Fig. 6. Aspergillus niger. Longitudinal section of hyphal tip. Filamentous mitochondria (M) and groups of ribosomes (R) stop short of the spitzenkörper region, which contains many vesicles (Vs). Similar vesicles are present at lower frequency more than I $2 \mu$ from the apex. The plasma membrane (P) presents a papillate outline more markedly at the apex than in non-extending regions. $\mathrm{CW}$, cell wall. Electron micrograph, glutaraldehyde-OsO ${ }_{4} \cdot \times 19,000(\mathrm{I} 0,000 \times \mathrm{I} \cdot 9)$. 

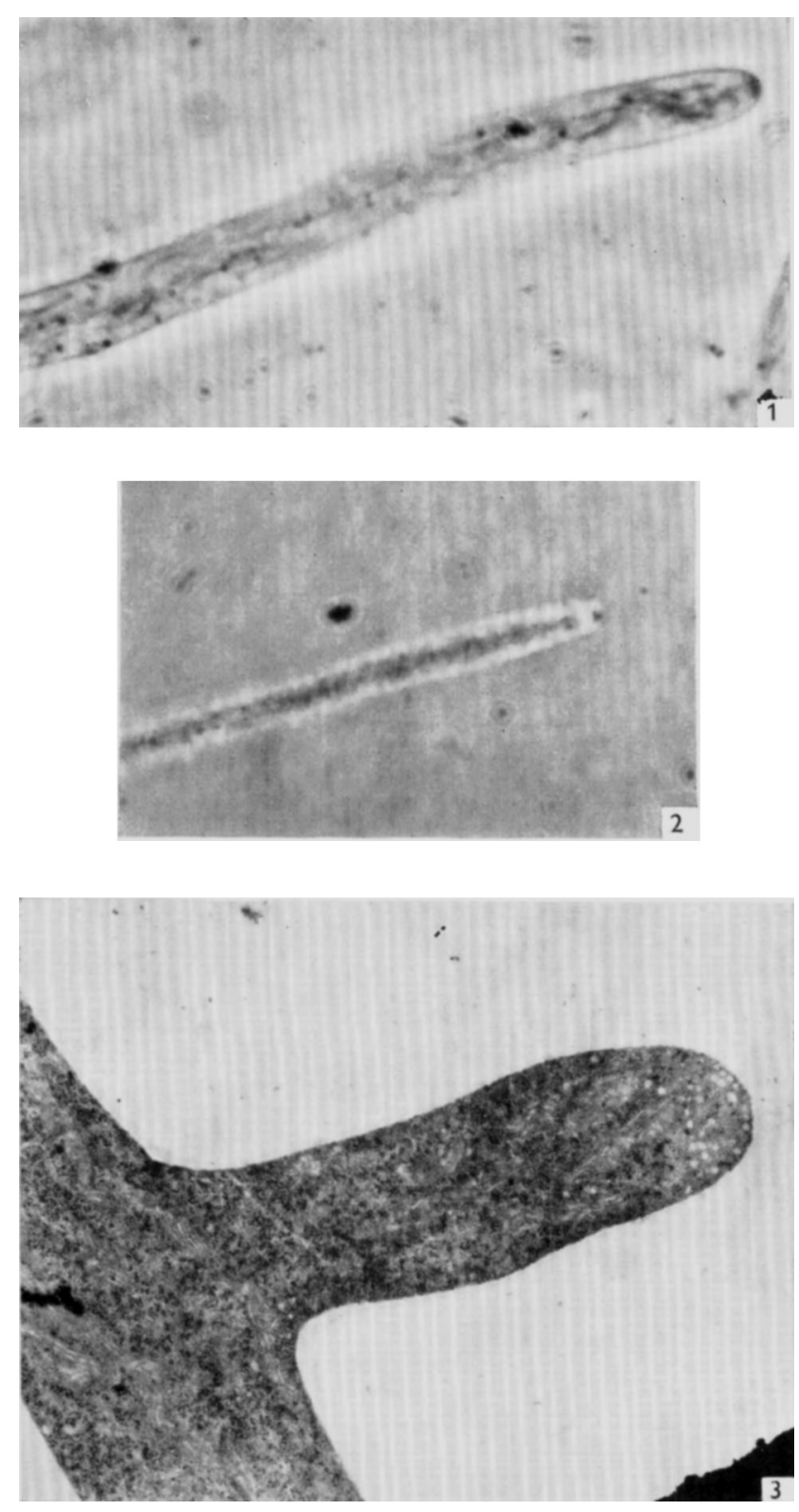


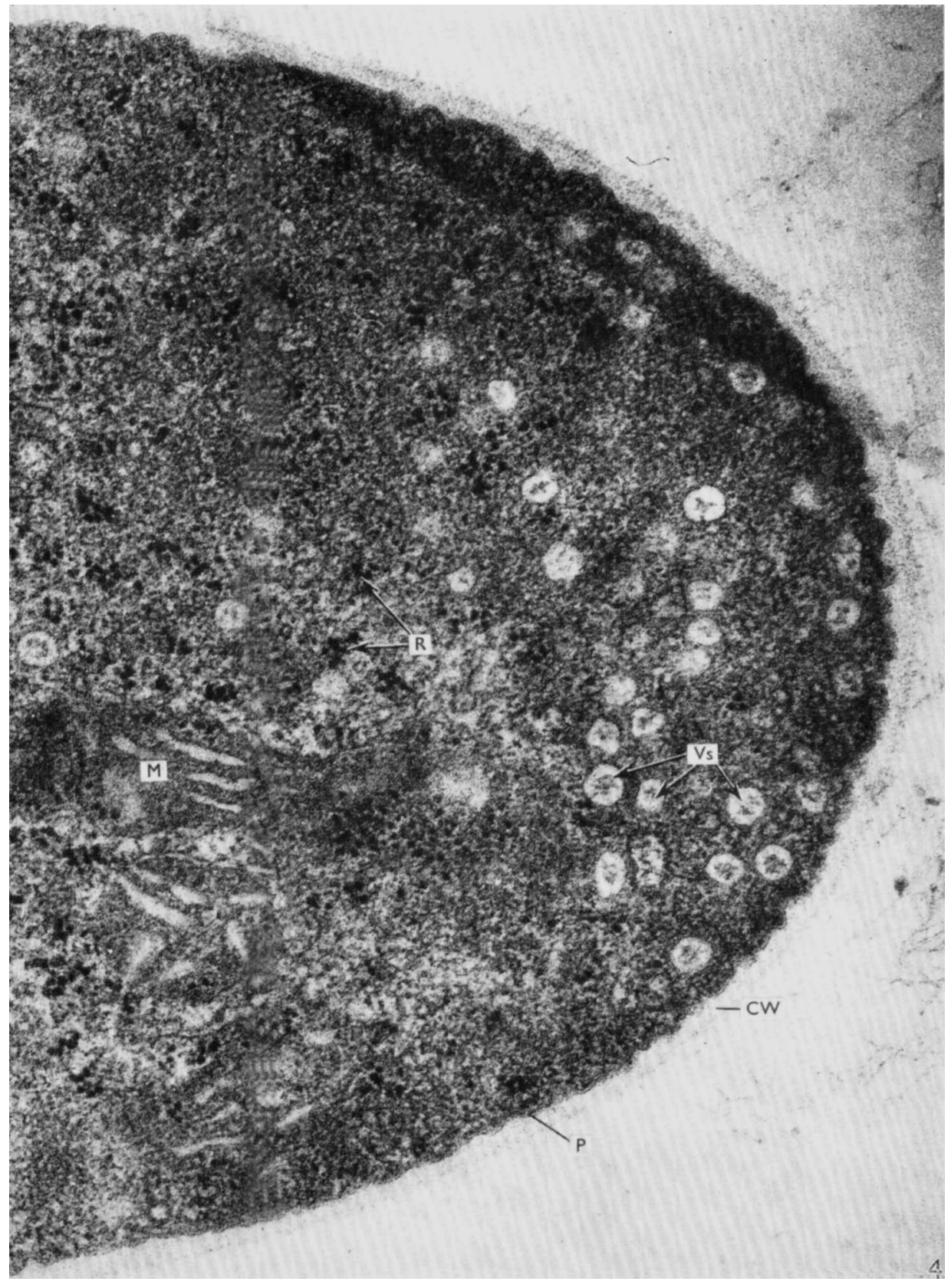

W. K. MCCLURE, D. PARK AND P. M. ROBINSON 


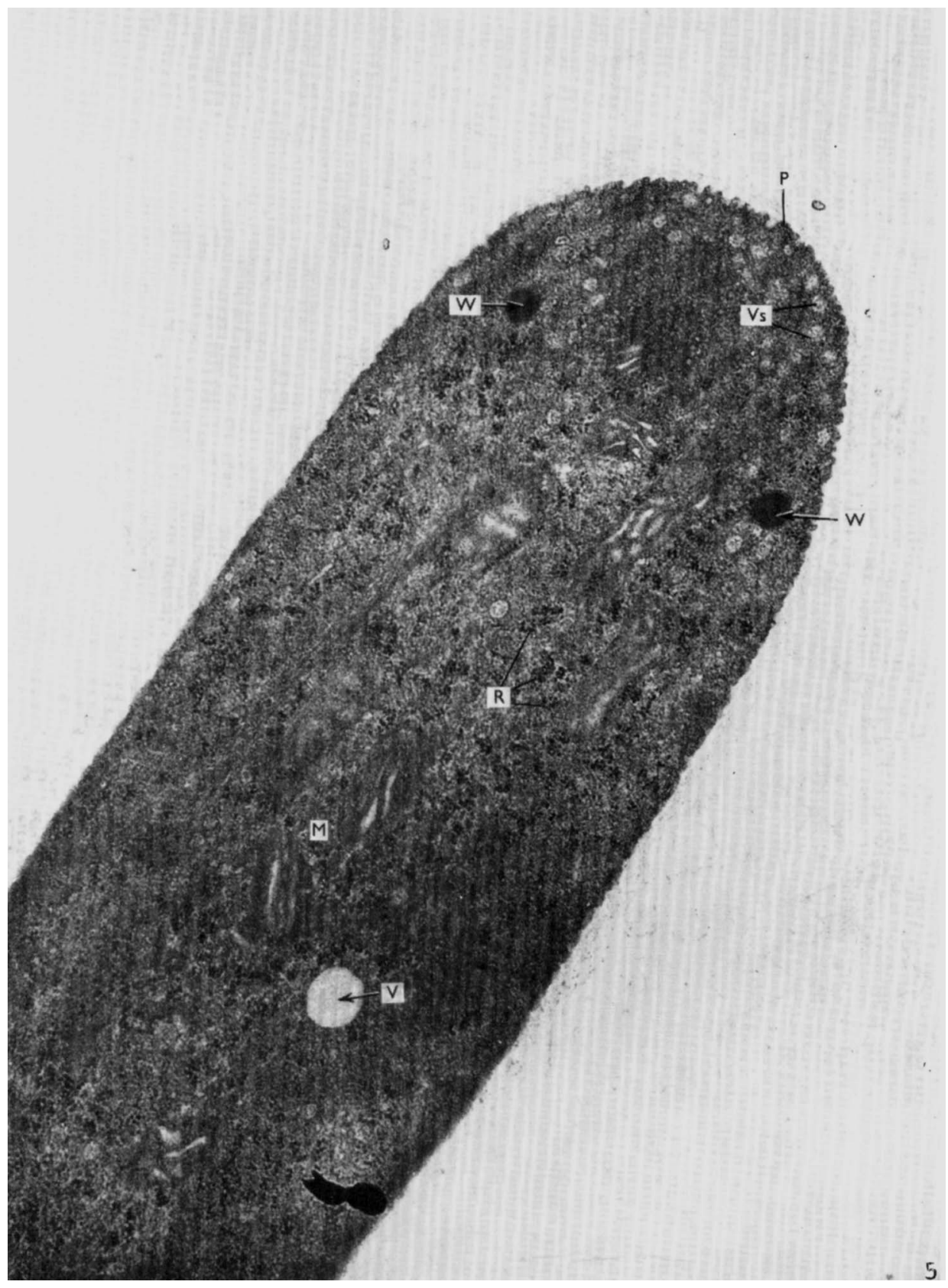

W. K. MCCLURE, D. PARK AND P. M. ROBINSON 
Journal of General Microbiology, Vol. 50, No. 2

Plate 4

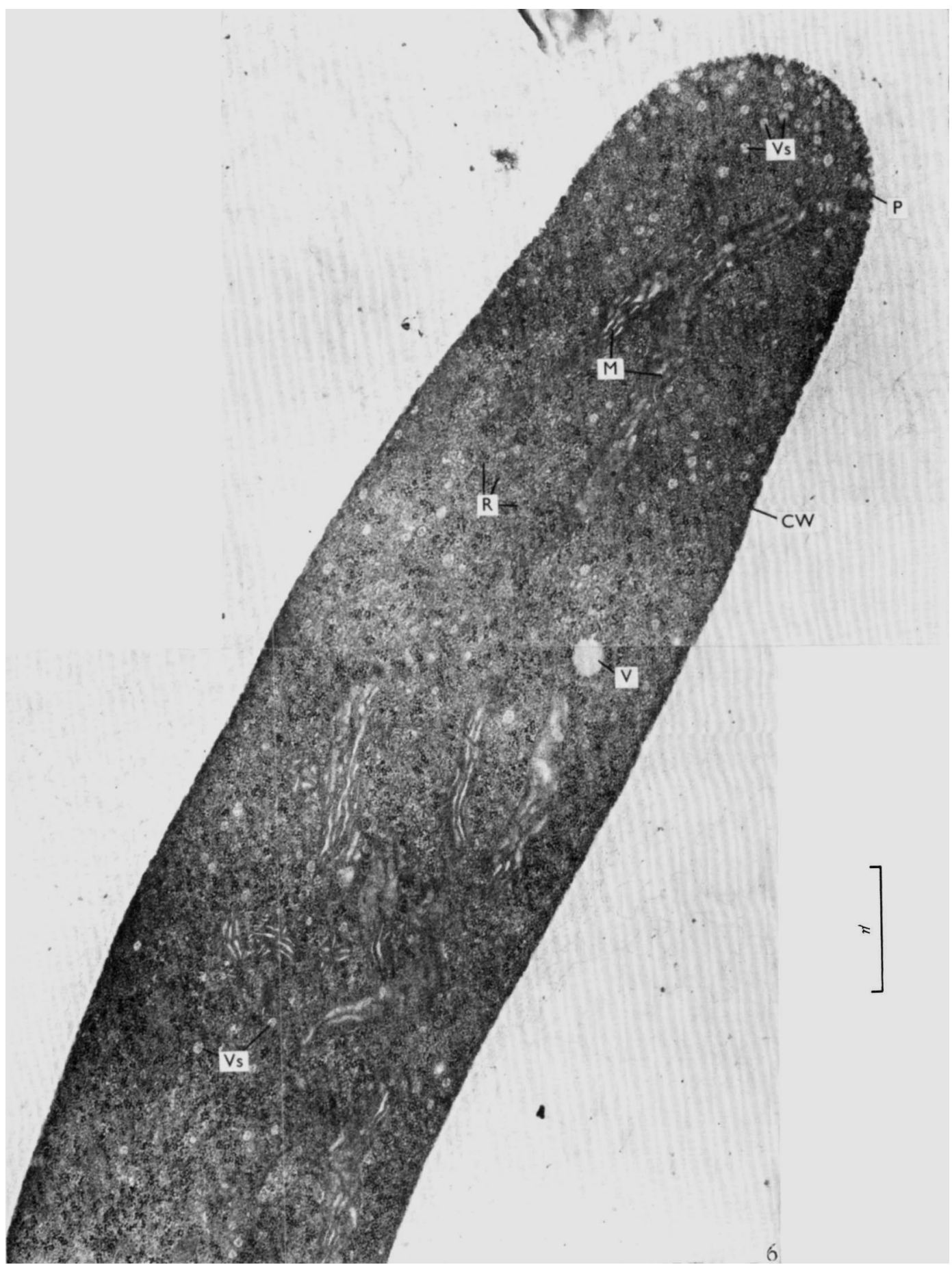

W. K. MCCLURE, D. PARK AND P. M. ROBINSON 